\title{
Neutron spin structure results from JLab Hall A
}

\author{
Zein-Eddine Meziani
}

Department of Physics, Temple University, Philadelphia, PA 19122

\begin{abstract}
My presentation will focus on some of the latest results of the neutron spin physics program at Jefferson Laboratory in Hall A using a polarized ${ }_{3} \mathrm{He}$ target. This program includes several completed experiments in which the spin structure functions of $3 \mathrm{He}$ were measured. The covered kinematic regions were these measurements were performed include the low $Q_{2}$ resonance and inelastic regions and the high $Q_{2}$ deep inelastic region. These experiments offer a ground for testing our understanding of the strong regime of quantum chromodynamics (QCD) through the determination of the neutron spindependent structure functions and their moments.
\end{abstract}

\section{INTRODUCTION}

After 25 years of spin structure measurements at the high energy physics laboratories (i.e. CERN, SLAC and DESY) leading to the determination of the quark spin content of the nucleon [1,2] and culminating with the test of the Bjorken sum rule [3], the spin structure of the neutron in the deep inelastic large $x$ region at $Q_{2}$ (above $1 \mathrm{GeV}_{2}$ ) is still poorly measured. This region does not contribute much to the first moment of spin structure functions, but it is crucial for evaluating higher moments of these functions. These moments offer a testing ground of QCD because they are connected with specific matrix elements $[4,5]$ that are directly calculable in lattice QCD [6]. The spin structure function in this valence quark region can also be tested through quark model calculations. At momentum transfers below $Q_{2}=l \mathrm{GeV}_{2}$ the large $x$ region is dominated by the resonance contributions and information on the neutron spin structure is also very scarce. This region is needed to determine the higher twists corrections in deep inelastic scattering (DIS) using the operator product expansion (OPE) technique to express moments of structure functions. Here we present results of a precision measurement (JLab E99-117) of the neutron asymmetry $A_{1}^{n}$ in the valence region (large $x$ and $<22$ ). We also show results of moments of the neutron spin structure $g_{2}$ (JLab E94-010) at $<22^{\prime}$ s ranging from $1 \mathrm{GeV}_{2}$ to $0.1 \mathrm{GeV}_{2}$. The above experiments share the same experimental setup. They were carried out at Jefferson Lab in Hall A using a highly polarized electron beam (70$80 \%$ ) with an average current up to $15 \mu \mathrm{A}$ and a high pressure polarized (on average between $30 \%$ and $40 \%$ in-beam) ${ }_{3} \mathrm{He}$ target with the highest polarized luminosity in the world. Details on these experiments can be found at[7].

\section{SPIN AND FLAVOR DECOMPOSITION IN THE VALENCEQUARK REGION}

The virtual photon-nucleon asymmetry ${ }_{1}^{n}$ and spin structure function $g *\{$ are the most poorly known in the valence quark region $(x>0.3)$. This shortcoming is due to the small scattering cross sections at large* and $Q_{2}$ combined with a lack of high polarized luminosity facilities. This region, however, is clean and unambiguous since it is not polluted by sea quarks and gluons offering thus a unique opportunity to test predictions that are difficult if not impossible at low $x$. The set of predictions of $A_{1}^{n}$ in the valence quark region fall into two categories, those of RCQM's which break SU(6) symmetry in the ground state wave function by hyperfine interaction $[8,9]$, and those of pQCD with a hadron helicity conservation (HHC) constraint $[10,11]$ as $x \rightarrow 1$ which break SU(6)symmetry dynamically. The difference between these approaches is dramatic when the constituents flavor spin decomposition is performed. For a proton and in the case of pQCD with HHC, we have $\Delta \mathrm{u}(\mathrm{x}) / \mathrm{u}(\mathrm{x}) \rightarrow 1$ and $\Delta(\mathrm{x}) / \mathrm{d}(\mathrm{x}) \rightarrow 1$, while for the case of 
RCQM's $\Delta \mathrm{u} / \mathrm{u} \rightarrow 1, \Delta \mathrm{d} / \mathrm{d} \rightarrow-2 / 3$. We notice that in leading order $\mathrm{pQCD}$ with HHC $\Delta \mathrm{d} / \mathrm{d}$ changes sign from negative at low $x$ to positive at large $x$.
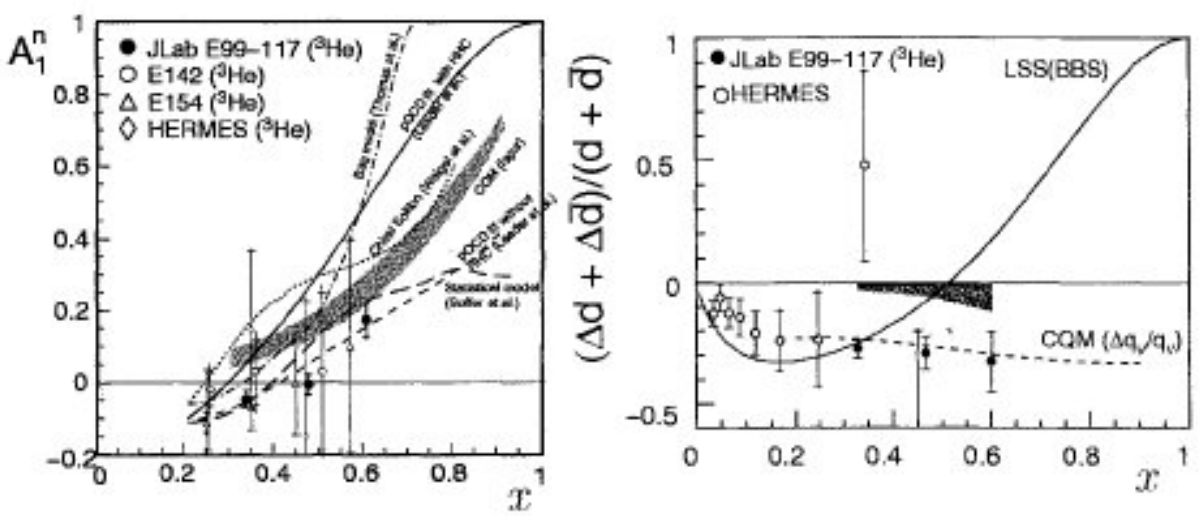

FIGURE 1. Left panel: Preliminary results of Jefferson Lab experiment E99-117 (solid circles) along with the world data (open symbols). The curves are predictions described in the text. Right panel: Spin flavor dependent down-quark distribution extracted from this experiment. The light error band is an estimate of the difference between the valence and the total down-quark distribution including the sea.

In Figure 1 (left panel) we show preliminary results of $A_{1}^{n}$. The first data point at $x=0.33$ is in good agreement with previous measurements. The data points show a clear change of sign of ${ }^{A_{1}^{n}}$ as $x$ increases and are compared with theoretical predictions. The total error in each point is dominated by the statistical error. The solid line is a prediction using HHC based on LSS(BBS) parameterization of $g_{1}^{n} / F_{1}^{n} \quad$ [12], the long dashed line is a prediction of $g_{1}^{n} / F_{1}^{n}$ from LSS 2001 parameterization at $\mathrm{Q}_{2}=5 \mathrm{GeV}_{2}$ without $\mathrm{HHC}$ constraints [13], The shaded area is a range of predictions of A" from the constituent quark model [9] while the dashed line is a calculation of the statistical model at $\mathrm{Q}_{2}=4 \mathrm{GeV}_{2}$ by Bourrely et. al. [14]. The short-dashed line is the chiral soliton model prediction at $\mathrm{Q}_{2}=3 \mathrm{GeV}_{2}$ by Weigel, Gamberg and Reinhardt [15]. Finally, the dot-dashed line is a bag model calculation but without meson cloud by Boros and Thomas [16] Data from Hermes and SLAC are original values without being re-analyzed for the A contribution of the nuclear corrections. We used the quark parton model interpretation of $g_{l}$ and $F_{l}$ to perform a flavor decomposition of the spin dependent quark distributions assuming a negligible strange quark contribution above $x=0.3$. The down-quark distribution obtained in E99-117 (filled squares) along with preliminary results of the HERMES semi-inclusive measurements (open circles) [17] are also shown in Fig. 1 (left panel). The solid line is a pQCD fit to the world data using the HHC constraint as $x->1$. The dashed line correspond to an RCQM prediction. It is clear that up to $x=0.6$ the data favor the RCQM rather than the HHC pQCD based calculations. In the latter no orbital angular momentum (OAM) in considered while in the RCQM some OAM is included through the small components of the nucleon wavefunction. These results, perhaps point towards the importance of considering the orbital momentum or quarks in the nucleon wave function. In the meantime, one has to wait for a more complete QCD calculation.

\section{BURKHARDT-COTTHIGHAM SUM RULE AND HIGHER MOMENTS OF STRUCTURE FUNCTIONS}

The $\mathrm{g}_{2}$ structure function obeys the Burkhardt-Cotthigham sum rule 


$$
\Gamma_{2}\left(Q^{2}\right)=\int_{0}^{1} g_{2}\left(x, Q^{2}\right) d x=0
$$

which was derived from the dispersion relation and the asymptotic behavior of the corresponding Compton amplitude [18]. This sum rule is true $t$ all $Q_{2}$ and does not follow from the OPE. It is rather a super-convergence relation based on Regge asymptotics as discussed in the review paper by Jaffe [19]. Many scenarios which invalidate this sum rule have been discussed in the literature. Surprisingly a first precision measurement of $£_{2}$ of the proton at SLAC at $Q_{2}=5 \mathrm{GeV}_{2}$ but with a limited range of $x$ has revealed a deviation of this sum rule for the proton at the level of three standard deviations [20]. In contrast, the neutron sum rule is poorly measured but consistent with zero at the one standard deviation. IN Fig. 2 (left panel) we show $\Gamma_{2}^{n}$ in the measured region (solid circles) and after adding the elastic contribution evaluated using Mergell et al. parameterization of $G_{M}^{n}$ and $G_{E}^{n}$ [22] (open circles). The solid line is the resonances contribution evaluated using MAID [21]. The positive light grey band corresponds to the total experimental systematic error while the dark negative band is an estimated deep inelastic contribution (DIS) assuming $g_{2}=g_{2}^{W W}$ following the same method as in [23]. The data show that the $\mathrm{BC}$ sum rule is verified within uncertainties over the $Q_{2}$ range measured. Our result is at odds with the observed violation of this sum rule for the proton at high $Q_{2}$, where the elastic contribution is negligible. The neutron result of SLAC E155x $\lceil 20\rceil$ is consistent with the sum rule but with a rather large error bar.
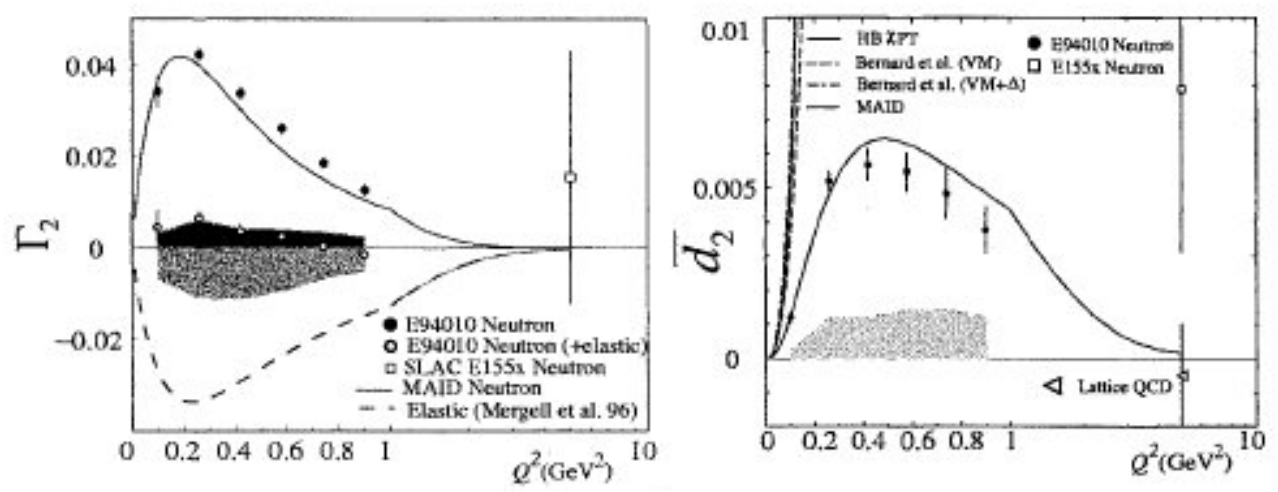

FIGURE 2. Left panel: Preliminary results of the $Q_{2}$ evolution of F? from JLab experiment E94-010 along with the world data from DIS and theoretical calculations (see text). Right panel: Preliminary results of $d_{2}^{n}$ compared to SLAC E155X results and $\chi \mathrm{PT}$ prediction at low Q2 and Lattice QCD calculation at $Q_{2}=5 \mathrm{GeV}_{2}$. (see text).

In the high $Q_{2}$ regime, the quantity

$$
d_{2}\left(Q^{2}\right)=\int_{0}^{1} x^{2}\left[2 g_{1}\left(x, Q^{2}\right)+3 g_{2}\left(x, Q^{2}\right)\right] d x,
$$

coincides with a twist three-matrix element and is related to the electric and magnetic polarizabilities of the color field [2]. At low $Q_{2}$, a region covered by our data, its conventional interpretation in terms of higher twist is not obvious. However a interpretation in terms of the electromagnetic polarizabilities is possible [24]. These polarizabilities can be evaluated using chiral perturbation theory $(\chi \mathrm{PT})$. Thus, the $Q_{2}$ evolution of $\mathrm{d}_{2}$ is a quantity that offers a dual interpretation depending on the resolution of the probe. At low $Q_{2}$ it is sensitive to the a direct polarization of the electromagnetic field present in the nucleon while at high $Q_{2}$ it is sensitive to the polarization of the color field in the nucleon. 
In Fig. 2 (right panel), $\bar{d}_{2}$ where the integration in equation (2) excludes the elastic peak is shown at several values of $Q_{2}$. The results of this experiment are the solid circles and the grey band represents their corresponding systematic uncertainty. The SLAC E155x neutron (open square) results is also shown. The solid line is the MAID calculation [21] while the solid line is a Heavy Baryon $\chi \mathrm{PT}$ calculation [24] and the covariant $\chi \mathrm{PT}$ [25]. The Lattice prediction [6] at $Q_{2}-5 \mathrm{GeV}_{2}$ for the neutron $\mathrm{d}_{2}$ matrix element is negative but close to zero. We note that all models (not shown here) predict $d_{2}^{n}$ to be negative or zero at large $Q_{2}$. At moderate $Q_{2}$ the data of E94-010 show a positive ${ }^{d_{2}^{n}}$ but decreasing perhaps to zero at high $Q_{2}$. The SLAC data also show a positive $d_{2}^{n}$ value but with a rather large error bar. More measurements are needed to have a complete determination of the transition from low to very high $Q_{2}$ of this important quantity.

\section{CONCLUSION}

In summary, we took advantage of the highly polarized beam and high pressure polarized $3 \mathrm{He}$ target at Jefferson Lab Hall A to investigate the internal spin structure of the neutron in the perturbative and the strong regimes of QCD. In E99-117 we have determined the world most precise down-quark helicity distribution in the valence region. The results agree with the constituent quark model rather than the HHC constrained pQCD prediction and perhaps point to the importance of the quark OAM in this region. In experiment E94010 we measured both $g_{1}$ and $g_{2}$ in the resonances region. We find the $\mathrm{BC}$ sum rule to be verified within uncertainties. The quantity $\bar{d}_{2}^{n}$ related to the electromagnetic polarizabilities at low $Q_{2}$ and the twist-three matrix element at large $Q_{2}$ was also measured and found to be small but finite.

\section{ACKNOWLEDGMENTS}

The work presented here was supported in part with funds provided to the Nuclear and Particle group at Temple University by the U.S. Department of Energy (DOE) under contract number DE-FG-02-94ER40844. The Southeastern Universities Research Association operates the Thomas Jefferson Accelerator Facility for the DOE under contractDE-AC05-84ER40150

\section{REFERENCES}

1. Hughes, E. W. and Voss, V, Ann. Rev. Nucl. Part. Sci. 49, 303 (1999).

2. Filippone, B. W. and Ji, X., Adv. in Nucl. Phys. 26,1 (2001).

3. Bjorken, J. D. Phys. Rev. 148, 1467 (1966); Phys. Rev. D 1,1376 (1970).

4. Shuryak, E.V. and Vainshtein, A.I., Nucl Phys'lfil, 141 (1982).

5. Jaffe, R. and Ji, X. Phys. Rev. Lett. 67, 552 (1991).

6. Gockeler, M., et al., Phys. Rev. D 63, 074506 (2001).

7. Details of experiments at wwwjlab.org/e991177 and www.jlab.org/e94010/.

8. Close, F. and Thomas, A. W., Phys. Lett. 6212,227(1988).

9. Isgur, N., Phys. Rev. D 59,034013 (1999).

10. Farrar, G. R. and Jackson, A. D., Phys. Rev. Lett. 35, 1416 (1975).

11. Brodsky, S. J., Burkhardt, M., Schmidt, I., Nucl Phys. B 441, 197 (1995).

12. Leader, E., Sidorov, A. V. and Stamenov, D. B., Int. J. Mod. Phys. A13, 5573 (1998).

13. Leader, E., Sidorov, A. V. and Stamenov, D. B., Eur. Phys. J. C 23,479 (2002).

14. Bourrely, C. Soffer, J. and Bucella, F., Eur. Phys. J. C23,479 (2002).

15. Weigel, H. and Gamberg, L., Nuc. Phys. A 680,48 (2000) and references therein.

16. Boros, C. and Thomas, A. W., Phys. Rev. D60, 074017 (1999)

17. Wendland, J., http: //hermes .desy.de/notes/pub/TRANS/Deltaq. 5p.ps . 
18. Burkhardt, H. and Cottingham, W. N., Ann. Phys. 56,453 (1970).

19. Jaffe, R., Comments Nucl. Part. Phys. 19, 239 (1990)

20. SLAC E155x, Anthony, P. L., et. al, Phys. Lett. B553,18 (2003).

21. Drechsel, D., Kamalov, S. and Tiator L., Phys. Rev. D 63, 114010 (2001).

22. Mergell, P., Meissner, Ulf-G. and Drechsel, D., Nucl Phys. A 596, 367 (1996).

23. Wandzura, S. and Wilczek, F., Phys. Lett. B 72 195, (1977).

24. Kao, C. W., Spitzenberg, T. and Vanderhaeghen, M., Phys. Rev. D 67, 016001 (2003).

25. Bernard, V. Hemmert, T. and Meissner, Ulf-G., Phys. Lett. B 545,105 (2002). 